\title{
Acreditación de criterio estudiantes: ¿Pueden nivelarse los índices de autoevaluación de carrera a través de acciones de mejora aplicando tutorías?
}

\section{Criteria accreditation students: Can career self-assessment indexes be leveled through improvement actions by applying tutoring?}

Juan Emilio Balás León

Universidad Politécnica Salesiana, Ecuador

Andrés Chávez

Universidad Internacional del Ecuador, Ecuador

Alexander Pavel Cedeño Velasco

Universidad De Guayaquil, Ecuador

Autor para correspondencia: jbalas@ups.edu.ec; anchavezer@uide.edu.ec;

alexander.cedenove@ug.edu.ec

Fecha de recepción: 10 de Septiembre de 2017 - Fecha de aceptación: 15 de Noviembre de 2017

Resumen: Este aporte indica en sus principios el contenido de las evidencias que comprende el criterio estudiantes en el modelo de autoevaluación del CEAACES. Luego mencionamos la relevancia de las tutorías en las que siendo un derecho y servicio que la academia ofrece a los estudiantes, también es importante el papel de acompañamiento de los tutores en los diferentes procesos incluyendo el del trabajo de titulación. Para comprobar esta idea se realizó una revisión del alcance y procesos del indicador tutorías, se aplicaron criterios como los estudiantes que aprueban materias con el puntaje mínimo, tutores asignados por materia, estudiantes reprobados y horas asignadas en diferentes períodos académicos para establecer fundamentos que justifiquen las necesidades de tutorías en el modelo universitario ecuatoriano. Se ha utilizado a la carrera de Administración de Empresas de la UPS GYE en donde se realizó un trabajo de campo en donde se generaron estadísticas utilizando la plataforma Sistema Nacional de Admisión. Entre los principales resultados tenemos que la cantidad de tutores se reduce de un semestre a otro, los estudiantes tienen mayor índice de repitencia en las asignaturas de primer a cuarto semestre y de que a pesar que la cantidad de horas asignadas para tutorías incrementa, también aumenta el retiro estudiantil y disminuye la retención de las cohortes. Finalmente, proponemos acciones de mejora para controlar éstos índices por medio de proyecciones de los desertores en base a las notas después del primer examen, plantear estrategias de ayuda y recuperación de puntaje por medio de tutorías y evidenciar el cambio de mentalidad de estudiantes y docentes. Palabras Clave: indicador tutorías; criterio estudiantes; acciones de mejora; ups gye

Abstract: This contribution indicates in its principles the content of the evidence that includes the students criterion in CEAACES self-evaluation model. Then we mentioned the relevance of the tutorials in which being a right and service that the academy offers to the students, it is also important the role of accompaniment of the tutors in the different processes including the work of the degree. To verify this idea, a review of the scope and processes of the tutorials indicator was carried out, criteria such as students passing subjects with the minimum score, tutors assigned by 
subject, failed students and assigned hours in different academic periods were applied to establish foundations that justify the needs of tutoring in the Ecuadorian university model. It has been used to the career of Business Administration of the UPS GYE where a fieldwork was carried out where statistics were generated using the National Admission System platform. Among the main results we have that the number of tutors is reduced from one semester to another, the students have higher repetition rate in the subjects from first to fourth semester and that although the amount of hours assigned for tutoring increases, it also increases the student withdrawal and decreases the retention of cohorts. Finally, we propose improvement actions to control these indices by means of projections of the deserters based on the notes after the first exam, to propose strategies of help and recovery of scoring through tutorials and to show the change of mentality of students and professors.

Key words: indicator tutorials; students criterion; improvement actions; ups gye

\section{Introducción}

Dentro del modelo de acreditación impuesto por el Consejo de Evaluación, Acreditación y Aseguramiento de la Calidad de la Educación Superior encontramos al criterio estudiantes, que controla las tutorías, su planificación, asignación de horas, informes de ejecución y modelos de control (Ceaaces, 2017). De la aplicación de las mismas, nacen nuevos conceptos como tutores, perfil de egreso, tasa de aprobación, retención, titulación. Los diferentes Centros de Educación Superior del Ecuador necesitan implantar acciones de mejora en donde las tutorías no solamente acompañen a los estudiantes, sino que al mismo tiempo mejoren el acompañamiento de los tutores para de esta manera disminuir el retiro estudiantil, mejorar la aprobación de cada asignatura y mantener la retención de las cohortes. Este aporte busca señalar la relación que existe entre el indicador tutorías con criterios de investigación para establecer sus necesidades y proponer mejoras que nivelen índices planteados en el modelo de autoevaluación de Carreras. (Autmendi, 2003; Rodríguez, 2004; Terenzini, Springer, Pascarella, \& Nora, 1995)

\section{Justificación}

Las tutorías son un servicio y derecho de los estudiantes que aparecen en el actual modelo de educación superior, ayudan al estudiante a mejorar su desempeño académico, le permiten nivelarse, auto prepararse e incluso adelantar materia. Las mismas se presentan a lo largo de su carrera, la Academia las toma en cuenta para sacar otros estudios como estudiantes reprobados por profesor y por materia. Este estudio es importante porque no basta simplemente con asignar horas de tutoría, debe determinarse su efectividad, conocer si éstas ayudan a controlar el retiro estudiantil y el grado en que se maneja la tasa de aprobación de cada asignatura (Alcon, 2003; Alonso, 2000; Raga, 2003, García, 1996).

\section{Objetivos}

\section{Objetivo General}

Conocer el alcance y necesidades del indicador tutorías en el modelo de acreditación del criterio estudiantes para establecer acciones de mejora que nivelen los índices de autoevaluación.

\section{Objetivos Específicos}


- Realizar una revisión de conceptos, alcance y procesos del indicador tutorías.

- Aplicar criterios de investigación para establecer fundamentos que justifiquen las necesidades de tutorías en el modelo universitario.

- Proponer acciones de mejora que conjunto a las tutorías controlen la tasa de deserción y retención estudiantil

\section{Marco Teórico}

Anteriormente se pensaba en la educación tradicional que vivió el Ecuador hasta principios del año 2000 en que mejor profesor universitario resultaba aquel que generaba mayor cantidad de reprobados. Dicho modelo no consideraba análisis complementarios que resultan de mejorar la eficiencia educativa, muchas carreras en los actuales momentos se preocupan no solamente de que los estudiantes se matriculen, paguen sus estudios, la academia necesita que estos estudiantes que empiezan su vida académica continúen todo el proceso en la mayor cantidad de inscritos posibles. De ahí salen términos como cohorte, tasa de aprobación, índice de repitencia, deserción estudiantil, tasa de titulación y retención respectivamente.

La tasa de aprobación es conocer la cantidad de estudiantes que aprueban cada asignatura. Mantiene conexión con aquellos que se retiran y con las exigencias de cada Universidad y de las acciones docentes. No se puede cambiar el hecho de que cada materia presenta diferente nivel de rigor. Más aún que cada docente presenta sus propias reglas de conducta, entrada - salida del aula, metodología, estas circunstancias pueden influir positiva o negativamente en la misma. (Balás, 2016)

Las tutorías poseen un acompañamiento por parte de los tutores asignados, en dicha posición se busca mejorar la eficiencia terminal, la Academia busca que a través de este proceso los estudiantes se preparen no solamente antes de examen, sino de manera continua en todo el proceso para de esta manera cuando lleguen a la unidad de titulación cumplan todos los lineamientos de su perfil de egreso.

Como menciona (Hower, 2007) un perfil de egreso contiene la descripción de los rasgos y competencias propias de un profesional que se desempeña en el ámbito de la sociedad, en campos que le son propios y enfrentando problemas, movilizando diversos saberes y recursos de redes y contextos, capaz de dar razón y fundamentación de sus decisiones y haciéndose responsable de sus consecuencias

La Carrera de Administración de Empresas de la Universidad Politécnica Salesiana Sede Guayaquil, como cualquier otro centro de educación superior trabaja de la manera presencial semestral. Dicho situación conlleva inconvenientes económicos debido a que en el primer semestre que empieza en Mayo y termina en Septiembre la cantidad de estudiantes inscritos es mucho mayor que en el semestre final que empieza en Octubre y termina en Febrero, efecto de esto es la variación de estudiantes y docentes, en el segundo semestre existe mayor cantidad de feriados que de una u otra manera si bien es cierto que permiten descansar, obligan a los docentes a tratar de cumplir en plan de clases más rápido y por consiguiente evaluar más severamente a los estudiantes. 
El abandono académico es inevitable, son circunstancias tanto personales, académicas y laborales que las originan. No se puede mencionar con seguridad el verdadero culpable, ya que algo de culpa posee la academia, el docente y el mismo estudiante. Las universidades piensan que la deserción es culpa del cuerpo docente, cuando les conviene olvidar que hay deficiencias en los procesos administrativos como factores de deserción. Los estudiantes siempre son los últimos en enterarse en el cambio de las normas administrativas. (Balás M. A., 2016)

También debemos mencionar que las autoridades máximas de la UPS realizan seguimientos para determinar bajo qué circunstancias los estudiantes se retiran, se les hace difícil aprobar una materia y bajo qué circunstancias se titulan. La gestión de tutorías tiene mucha relevancia para los implicados: autoridades, docentes y estudiantes porque a través de las mismas se pueden controlar las diferentes tasas mencionadas anteriormente.

De acuerdo a (Ceaaces, 2017) El indicador tutorías evalúa que la carrera asigne y de seguimiento a las tutorías ejecutadas por los profesores a los estudiantes. El período de evaluación corresponde a los dos últimos períodos académicos ordinarios o al último año concluido, antes del inicio del proceso de evaluación.

Como aporte personal, indicamos que el universitario proviene de un ambiente pedagógico manifestado en el Colegio o Unidad educativa en el cual se gradúa, estas instituciones debido a las reformas que les imponen los diferentes distritos han eliminado la cantidad de horas de materias esenciales, y es así que asignaturas como Matemáticas, Contabilidad y Estadística que se convierten en materias que constituyen un obstáculo y punto de inicio para desertar, razón por la cual en las universidades reina el modelo andragogico, educación de adultos

Tan visible es el efecto de la Andragogía, en la que el estudiante descubre que aunque su colegio pertenezca a un buen ranking, siempre existirá un vacío o tema que no pudo cubrir, la UPS oferta el servicio de tutorías que puede ser con el mismo docente o con cualquier otro de las áreas. Una de las limitancias es que se ven temas específicos y son realizadas en los cubículos de los docentes, en algunos casos los profesores asignados son toda la planta de tiempo completo y en casos mínimos los medios tiempos.

Andragogía es la ciencia que estudia el proceso de cómo los adultos aprenden y por lo tanto es un instrumento del cual se vale el estudiante adulto para construir un sistema de conocimientos que le permite incorporar nuevos aprendizajes prácticos y útiles necesarios para el desempeño eficiente de su profesión. (Aguilar, 2014)

Las experiencias reflejadas en la metodología aplicada indican que desde el primer semestre hasta el cuarto semestre en que aproximadamente están a mitad de la carrera, es donde necesitan mayor uso de tutorías porque el peso de las materias base de la carrera incluyen temas que debieron tratarse en Colegio, razón por la cual nace la necesidad de tutorías. En los últimos niveles, los estudiantes tienen que cumplir actividades adicionales como extensiones, prácticas profesionales, pasantías, cursos de Inglés y Computación, además si se considera que pueden trabajar, ser cabeza de familia y otro tipo de obligaciones, esto hace que disminuya el uso de tutorías para los niveles mencionados. 
El indicador tutorías tiene relación directa con la tasa de retención, la UPS apuesta fuertemente a que el continuo uso de tutorías eviten la deserción estudiantil, incluso para cualquiera de las modalidades de titulación, las tutorías aparecen para asegurar y complementar la cantidad de horas del trabajo final de graduación.

La tutoría de los trabajos de titulación es el proceso de acompañamiento que deberá otorgarse al estudiante durante su elaboración. En consecuencia, la orientación de la tutoría debe garantizar la validación de las capacidades cognitivas que se declaran en el perfil de egreso y que se evidencian en la profundidad y consistencia del trabajo de titulación, pueden ser presenciales y virtuales, individuales, grupales y en plenaria según la parte del trabajo que se está realizando, sus niveles de profundización y de necesidad de acompañamiento que tienen los estudiantes.

(CES, 2007)

\section{Tipos de tutorías pedagógicas}

Directas. - son aquellas que fueron designados tutores pedagógicos a docentes en su carga horaria porque generalmente dominan materias de ciencias duras como matemáticas aplicadas, matemáticas financieras, metodología de investigación, contabilidad general y de costos que son las que mayormente tiene incidencia de problemas, motivos porque los primeros niveles vienen estudiantes de colegio que pueden venir con deficiencias u otro factor la adaptabilidad a las metodologías de aprendizaje universitario.

Se dan a nivel individual o grupal, todas son registradas o anunciadas al gestor para luego la coordinación del mismo, este a su vez presentará las evidencias de realizado y se hará el monitoreo del estudiante sobre su evolución.

Indirectas. - Aquellas que se generan cuando en el cuadro de estadísticas de asignatura salgan con porcentaje de riesgo, por tal razón el tutor pedagógico puede asistir a la materia para ser mediador donde pregunta a los estudiantes dentro del programa de estudio si tiene falencias para que el profesor en conjunto con el tutor se programe la tutoría de refuerzo permitiendo con esto que se entienda mejor los temas a tratar.

\section{Metodología}

El centro de Educación Superior objeto de estudio es la carrera de Administración Sede Guayaquil de la UPS GYE, se realizará un trabajo de campo, en donde por medio de la información brindada de la Secretaria del Campus Centenario Guayaquil, junto a datos de la plataforma informática SNA - Sistema Nacional de Admisión se analizarán la cantidad de tutores asignados por cada semestre realizando una comparación de Mayo 2017 a Febrero 2018. Posteriormente se obtendrán criterios para determinar las necesidades de tutoría teniendo en cuenta que se ofertan 2 mallas, que son la rediseñada y unificada. Los criterios que se aplicarán son estudiantes reprobados por materia y estudiantes que aprueban con puntaje mínimo. Seguido de esto nos enfocamos a ver la evolución de las horas asignadas semanalmente en tutorías y finalmente se estudia la tendencia de la tasa de deserción y de retención para proponer acciones de mejora que permitan un mejor manejo de la eficiencia terminal. 


\section{Resultados}

\section{Tutores Asignados Mayo 2017 a Febrero 2018}

\begin{tabular}{lc} 
Tabla 1: Primer Semestre - Mayo a Octubre/2017 \\
\hline Materia & Tutores Asignados \\
\hline Fundamentos De Administración & 1 \\
Fundamentos Contables & 4 \\
Metodología De La Investigación & 2 \\
Matemáticas & 2 \\
Comunicación Oral Y Escrita & 4 \\
Total Tutores Primer Semestre & 13 \\
\hline
\end{tabular}

Elaborado por: Los autores

La Carrera de Administración de Empresas UPS Sede Guayaquil, empezó a ofertar su nueva malla de Licenciatura la cual empezó en Mayo/2017. Progresivamente se irán incrementando los nuevos cursos hasta desaparecer la última promoción de Ingenieros Comerciales.

Tabla 2: Segundo Semestre -Mayo a Octubre/2017

\begin{tabular}{lcc}
\hline Materia & $\begin{array}{c}\text { Tutores Asignados Mayo - } \\
\text { Septiembre }\end{array}$ & $\begin{array}{c}\text { Tutores Asignados Octubre - } \\
\text { Febrero }\end{array}$ \\
\hline Administración General Ii & 2 & 2 \\
Contabilidad Ii & 1 & 2 \\
Historia Y Filosofía De La & 2 & 1 \\
Ciencia & 2 & 2 \\
Informática Aplicada Ii & 3 & 1 \\
Introducción A La Economía & 2 & 2 \\
Matemática Aplicada Ii & 12 & 10 \\
Total Tutores Segundo & & \\
Semestre & & \\
\hline
\end{tabular}

Elaborado por: Los autores

Tabla 3: Tercer Semestre- Mayo a Octubre/2017

\begin{tabular}{lcc}
\hline Materia & $\begin{array}{c}\text { Tutores Asignados Mayo - } \\
\text { Septiembre }\end{array}$ & $\begin{array}{c}\text { Tutores Asignados Octubre - } \\
\text { Febrero }\end{array}$ \\
\hline Contabilidad De Costos I & 3 & 3 \\
Espiritualidad Juvenil & 1 & 3 \\
Salesiana & 3 & 2 \\
$\quad$ Estadística Descriptiva & 3 & 3 \\
Matemática Aplicada Iii & 1 & 3 \\
Metodología De La & 3 & 2 \\
Investigación & 14 & 16 \\
Microeconomía & & \\
Total Tutores Tercer Semestre & &
\end{tabular}

Elaborado por: Los autores 
Tabla 4: Cuarto Semestre- Mayo a Octubre/2017

\begin{tabular}{lcc}
\hline Materia & Tutores Asignados Mayo - Septiembre & $\begin{array}{c}\text { Tutores Asignados Octubre - } \\
\text { Febrero }\end{array}$ \\
\hline Auditoría & 1 & 2 \\
Comportamiento Organizacional & 1 & 3 \\
Contabilidad De Costos Ii & 2 & 4 \\
Estadística Inferencial & 2 & 4 \\
Ingeniería Económica I & 2 & 5 \\
Macroeconomía & 1 & 4 \\
Total Tutores Cuarto Semestre & 9 & 22 \\
\hline
\end{tabular}

Elaborado por: Los autores

En el segundo y tercer semestre, la cantidad de tutores asignados disminuye debido a la reducción de estudiantes, mientras en el cuarto semestre en las materias de especialización como Costos II, Estadística Inferencial, Ingeniería Económica y Macroeconomía existe mayor cantidad de tutores debido a que las mismas generan alto grado de repitencia.

\section{Necesidades De Tutoría}

Como se ha mencionado la carrera objeto de estudio posee 2 mallas ofertadas, la rediseñada o nueva y la unificada o antigua. En ambos casos se determinará la cantidad de reprobados y las materias en las que los estudiantes pasan con el puntaje mínimo considerado de 70 a 73 puntos. Este análisis proviene de las notas finales del período de Mayo a Septiembre / 2017 en donde se aplicarán diferentes criterios para determinar las asignaturas que requieran mayor demanda de tutorías.

\section{Malla Rediseñada Criterio 1}

Tabla 5: Estudiantes Reprobados por Materia Malla Rediseñada

\begin{tabular}{clr}
\hline Puesto & \multicolumn{1}{c}{ Materia } & Cantidad \\
\hline 1 & Matemáticas & 111 \\
2 & Fundamentos Contables & 92 \\
3 & Fundamentos De Administración & 71 \\
4 & Metodología De La Investigación & 65 \\
5 & Comunicación Oral Y Escrita & 63 \\
Total Registros Reprobados Malla Rediseñada & 402
\end{tabular}

Fuente: Secretaria de Campus UPS Sede Guayaquil

\section{Malla Rediseñada Criterio 2}

Tabla 6: Estudiantes Aprobados con puntaje mínimo Malla Rediseñada

\begin{tabular}{clrrrrr}
\hline Puesto & \multicolumn{1}{c}{ Materia } & 70 & 71 & 72 & 73 & Cantidad \\
\hline 1 & Matemáticas & 39 & 14 & 13 & 9 & 75 \\
2 & Fundamentos Contables & 26 & 16 & 14 & 11 & 67 \\
3 & Comunicación Oral Y Escrita & 20 & 5 & 5 & 10 & 40 \\
\hline
\end{tabular}




\begin{tabular}{ccccccr}
\hline 4 & Metodología De La Investigación & 13 & 9 & 6 & 6 & 34 \\
5 & Fundamentos De Administración & 16 & 6 & 4 & 4 & 30 \\
Total Registro De Estudiantes Aprobados Con Puntaje Minimo Malla Rediseñada & & 246 \\
\hline
\end{tabular}

Fuente: Secretaria de Campus UPS Sede Guayaquil

En base a estos datos se recomienda asignar mayor número de tutorías a las asignaturas de Matemáticas y Fundamentos Contables en la malla rediseñada, cabe señalar que el Rectorado ha aprobado las tutorías personalizadas con ayudantes de cátedra en estas asignaturas.

\section{Malla Unificada Criterio 2}

Tabla 7: Estudiantes Reprobados por profesor Malla unificada

\begin{tabular}{rlr}
\hline Puesto & \multicolumn{1}{c}{ Materia } & Cantidad \\
\hline 1 & Microeconomía & 56 \\
2 & Matemática Aplicada Ii & 54 \\
3 & Matemática Aplicada Iii & 39 \\
4 & Estadística Descriptiva & 31 \\
5 & Estadística Descriptiva & 31 \\
6 & Administración General Ii & 31 \\
7 & Introducción A La Economía & 29 \\
8 & Contabilidad Ii & 28 \\
9 & Matemática Aplicada I & 28 \\
10 & Contabilidad De Costos I & 24 \\
11 & Informática Aplicada Ii & 23 \\
12 & Ingeniería Económica I & 20 \\
13 & Finanzas I & 20 \\
14 & Contabilidad I & 20 \\
15 & Estadística Inferencial & 18 \\
16 & Fundamentos De Marketing & 16 \\
17 & Informática Aplicada I & 15 \\
18 & Macroeconomía & 14 \\
19 & Metodología De La Investigación & 13 \\
20 & Espiritualidad Juvenil Salesiana & 13 \\
21 & Historia Y Filosofía De La Ciencia & 13 \\
22 & Contabilidad De Costos Ii & 12 \\
23 & Finanzas Ii & 10 \\
24 & Gestión De Empresas Iii & 10 \\
25 & Gerencia De Operaciones Ii & 9 \\
26 & Inglés De Negocios Ii & 7 \\
27 & Gerencia De Operaciones I & 7 \\
28 & Antropología Cristiana & \\
29 & Comercio Exterior & \\
30 & Ingeniería Económica Ii & \\
31 & Administración General I & \\
\hline & & \\
\hline
\end{tabular}




\begin{tabular}{|c|c|c|}
\hline 32 & Comportamiento Organizacional & 6 \\
\hline 33 & Técnicas De Expresión Oral Y Escrita & 6 \\
\hline 34 & Pensamiento Social De La Iglesia & 5 \\
\hline 35 & Inglés De Negocios I & 5 \\
\hline 36 & Gestión De Empresas Ii & 5 \\
\hline 37 & Ingeniería Financiera & 4 \\
\hline 38 & Administración De Recursos Humanos & 4 \\
\hline 39 & Sistemas De Información Gerencial & 4 \\
\hline 40 & Gerencia Estratégica & 4 \\
\hline 41 & Relaciones Económicas Internacionales & 4 \\
\hline 42 & Gestión De Empresas I & 4 \\
\hline 43 & Auditoría & 4 \\
\hline 44 & Investigación De Mercados & 4 \\
\hline 45 & Programación Básica & 4 \\
\hline 46 & Comercio Internacional & 3 \\
\hline 47 & Marketing Estratégico & 2 \\
\hline 48 & Ética & 2 \\
\hline 49 & Presupuestos I & 2 \\
\hline 50 & Derecho Empresarial I & 2 \\
\hline 51 & Finanzas Internacionales & 2 \\
\hline 52 & Finanzas Corporativas & 2 \\
\hline 53 & Mercado De Capitales & 2 \\
\hline 54 & Trámites Aduaneros & 2 \\
\hline 55 & Derecho Empresarial Ii & 2 \\
\hline 56 & Negociación & 1 \\
\hline 57 & Ecología Y Educación Ambiental & 1 \\
\hline 58 & Gerencia De La Calidad & 1 \\
\hline 59 & Gestión De Marketing & 1 \\
\hline 60 & Legislación Aduanera & 1 \\
\hline 61 & Administración De Ventas & 1 \\
\hline 62 & Promoción Y Publicidad & 1 \\
\hline 63 & Marketing Internacional & 1 \\
\hline \multicolumn{2}{|c|}{ Total Estudiantes Reprobados Mayo A Septiembre / 2017} & 753 \\
\hline
\end{tabular}

Fuente: Secretaria de Campus UPS Sede Guayaquil

\section{Malla Unificada Criterio 3}

Tabla 8: Estudiantes Aprobados con puntaje mínimo 70 a 73 en Malla unificada

\begin{tabular}{rlrrrrr}
\hline Puesto & \multicolumn{1}{c}{ Materia } & 70 & 71 & 72 & 73 & Cantidad \\
\hline 1 & Contabilidad Ii & 65 & 10 & 6 & 4 & 85 \\
2 & Estadística Descriptiva & 29 & 12 & 13 & 13 & 67 \\
3 & Contabilidad De Costos I & 21 & 7 & 15 & 19 & 62 \\
4 & Matemática Aplicada Ii & 43 & 5 & 9 & 5 & 62 \\
\hline
\end{tabular}




\begin{tabular}{|c|c|c|c|c|c|c|}
\hline 5 & Microeconomía & 23 & 17 & 13 & 8 & 61 \\
\hline 6 & Matemática Aplicada Iii & 32 & 10 & 5 & 7 & 54 \\
\hline 7 & Macroeconomía & 22 & 10 & 7 & 8 & 47 \\
\hline 8 & Estadística Inferencial & 36 & 3 & 5 & 3 & 47 \\
\hline 9 & Gestión De Empresas Iii & 18 & 5 & 13 & 9 & 45 \\
\hline 10 & Ingeniería Económica I & 18 & 16 & 3 & 5 & 42 \\
\hline 11 & Inglés De Negocios Ii & 25 & 9 & 5 & 3 & 42 \\
\hline 12 & Ingenieria Económica Ii & 15 & 8 & 8 & 10 & 41 \\
\hline 13 & Inglés De Negocios I & 13 & 11 & 6 & 9 & 39 \\
\hline 14 & Programación Básica & 13 & 9 & 7 & 9 & 38 \\
\hline 15 & Gestión De Empresas Ii & 19 & 9 & 4 & 5 & 37 \\
\hline 16 & Informática Aplicada Ii & 19 & 5 & 5 & 5 & 34 \\
\hline 17 & Finanzas I & 16 & 6 & 6 & 6 & 34 \\
\hline 18 & Introducción A La Economía & 10 & 7 & 8 & 5 & 30 \\
\hline 19 & Finanzas Corporativas & 4 & 5 & 9 & 10 & 28 \\
\hline 20 & Comercio Internacional & 14 & 2 & 4 & 5 & 25 \\
\hline 21 & Finanzas Ii & 4 & 7 & 3 & 10 & 24 \\
\hline 22 & Ingeniería Financiera & 5 & 6 & 6 & 6 & 23 \\
\hline 23 & Marketing Estratégico & 9 & 3 & 6 & 5 & 23 \\
\hline 24 & Gerencia Estratégica & 9 & 3 & 3 & 8 & 23 \\
\hline 25 & Historia Y Filosofía De La Ciencia & 10 & 2 & 3 & 8 & 23 \\
\hline 26 & Contabilidad De Costos Ii & 9 & 5 & 6 & 3 & 23 \\
\hline 27 & Presupuestos I & 9 & 3 & 4 & 6 & 22 \\
\hline 28 & Sistemas De Información Gerencial & 11 & 2 & 2 & 7 & 22 \\
\hline 29 & Comercio Exterior & 7 & 6 & 5 & 3 & 21 \\
\hline 30 & Metodología De La Investigación & 5 & 8 & 4 & 3 & 20 \\
\hline 31 & Mercado De Capitales & 4 & 7 & 4 & 4 & 19 \\
\hline 32 & Gerencia De Operaciones I & 8 & 1 & 5 & 4 & 18 \\
\hline 33 & Finanzas Internacionales & 6 & 5 & 2 & 5 & 18 \\
\hline 34 & Administración General Ii & 6 & 4 & 6 & 2 & 18 \\
\hline 35 & Gestión De Marketing & 5 & 6 & 4 & 2 & 17 \\
\hline 36 & Gerencia De Operaciones Ii & 5 & 3 & 5 & 4 & 17 \\
\hline 37 & Relaciones Económicas Internacionales & 3 & 5 & 4 & 3 & 15 \\
\hline 38 & Auditoría & 2 & 5 & 3 & 5 & 15 \\
\hline 39 & Informática Aplicada I & 8 & 3 & 2 & 2 & 15 \\
\hline 40 & Derecho Empresarial I & 8 & 0 & 3 & 3 & 14 \\
\hline 41 & Fundamentos De Marketing & 5 & 2 & 1 & 6 & 14 \\
\hline 42 & Gestión De Empresas I & 5 & 2 & 2 & 4 & 13 \\
\hline 43 & Espiritualidad Juvenil Salesiana & 4 & 2 & 5 & 1 & 12 \\
\hline 44 & Investigación De Mercados & 3 & 1 & 4 & 3 & 11 \\
\hline 45 & Gerencia De La Calidad & 0 & 6 & 3 & 1 & 10 \\
\hline 46 & Matemática Aplicada I & 2 & 5 & 2 & 1 & 10 \\
\hline 47 & Contabilidad I & 6 & 3 & 0 & 0 & 9 \\
\hline
\end{tabular}




\begin{tabular}{llrrrrr}
\hline 48 & Ecología Y Educación Ambiental & 2 & 4 & 1 & 1 & 8 \\
49 & Pensamiento Social De La Iglesia & 4 & 1 & 1 & 1 & 7 \\
50 & Trámites Aduaneros & 4 & 1 & 0 & 2 & 7 \\
51 & Promoción Y Publicidad & 2 & 4 & 0 & 1 & 7 \\
52 & Administración De Recursos Humanos & 0 & 3 & 1 & 2 & 6 \\
53 & Comportamiento Organizacional & 2 & 1 & 1 & 2 & 6 \\
54 & Técnicas De Expresión Oral Y Escrita & 2 & 1 & 2 & 1 & 6 \\
55 & Negociación & 3 & 0 & 0 & 2 & 5 \\
56 & Plan Anual De Mercadeo & 3 & 0 & 2 & 0 & 5 \\
57 & Promoción De Exportaciones & 1 & 0 & 2 & 1 & 4 \\
58 & Ética & 0 & 0 & 1 & 3 & 4 \\
59 & Gerencia Financiera & 2 & 0 & 2 & 0 & 4 \\
60 & Valoración Aduanera & 0 & 2 & 0 & 1 & 3 \\
61 & Gestión De Empresas Iv & 1 & 0 & 1 & 1 & 3 \\
62 & Negocios Internacionales & 1 & 0 & 0 & 1 & 2 \\
63 & Legislación Aduanera & 0 & 1 & 0 & 1 & 2 \\
64 & Derecho Empresarial Ii & 1 & 0 & 0 & 1 & 2 \\
65 & Antropología Cristiana & 2 & 0 & 0 & 0 & 2 \\
66 & Marketing Internacional & 0 & 0 & 2 & 0 & 2 \\
67 & Valoración De Empresas & 2 & 0 & 0 & 0 & 2 \\
68 & Auditoría Del Marketing & 0 & 0 & 0 & 1 & 1 \\
69 & Administración General I & 1 & 0 & 0 & 0 & 1 \\
70 & Administración De Ventas & 0 & 0 & 0 & 1 & 1 \\
71 & Gestión De Riesgo Financiero & 1 & 0 & 0 & 0 & 1 \\
72 & Derecho Empresarial Iii & 0 & 0 & 0 & 0 & 0 \\
& Regsitro De Aprobados Con Puntaje Mínimo Malla Unificada & & 1480
\end{tabular}

Fuente: Secretaria de Campus UPS Sede Guayaquil

Tomando en cuenta los datos presentados en la malla unificada, las asignaturas de segundo a cuarto nivel presentan mayor deficiencia académica, incluso en las materias de carácter espiritual, por lo que se necesita asignar mayor presencia de tutorías en las mismas. También se debe hacer un seguimiento a los docentes señalados que tienen mayor número de reprobados, si bien es cierto se desea mantener un status académico, muchos de estos colegas necesitan un momento de reflexión y cambio del modelo metodológico.

\section{Horas asignadas de tutorías Mayo/2016 a Febrero/2018}

Tabla 9: Comparativo de Horas de Tutorías

\begin{tabular}{lc}
\hline Período Académico & Horas semanales asignadas \\
\hline Mayo 2016 - Septiembre 2016 & 175 \\
Octubre 2016 - Febrero 2017 & 216 \\
Mayo 2017 - Septiembre 2017 & 215 \\
Octubre 2017 - Febrero 2018 & 232 \\
\hline
\end{tabular}

Elaborado por: Los autores 
La carrera de Administración de Empresas UPS GYE ha realizado las gestiones académicas respectivas para incrementar la cantidad de horas ofertadas de tutorías hacia sus estudiantes, tomando como base el primer semestre del año 2016 se inició con 175 horas semanales de tutorías que se ha ido incrementando como muestra la tabla 9 hasta llegar a la base actual del primer semestre del año 2017 que corresponde a 232 horas semanales.

\section{Tasa de retención y retiro estudiantil Carrera de Administración de Empresas Guayaquil.}

Tomando en cuenta el total de estudiantes inscritos en el Sistema Nacional de Admisión para determinar el nivel de retiro de cada cohorte esta cantidad se divide para aquellos estudiantes que han anulado las materias respectivas. Se toma como base el semestre de Octubre 2015 a Febrero 2016 hasta Mayo 2017 a Octubre 2017; se realiza el análisis tomando en cuenta un enfoque administrativo y otro académico, bajo este análisis a los estudiantes retirados se los denomina Nota 0 .

Analizando la parte Administrativa y Económica en el período 46 al 47 se registran una disminución de 337 no inscritos; del 47 al 48 aumentan los inscritos a 354; del 48 al 49 disminuyen los inscritos en 352 y del 49 al 50 la disminución es de 261 estudiantes.

En cuanto al retiro por parte académica, la tendencia muestra que desde Mayo / 2015 la cantidad de estudiantes que anulan materias y que contribuye a que se retiren aumenta considerablemente; de Mayo 2015 a Febrero 2016 el retiro aumenta 7\%; de Febrero 2016 a Septiembre 2016 aumenta 21\%; de Septiembre 2016 a Febrero 2017 aumenta 21\% y de Febrero 2017 a Septiembre 201750 disminuye el retiro al 3\%, pero no deja de ser preocupante.

Se puede deducir que la propuesta académica planteada por los máximos directivos de la UPS ha incidido en que se rebaje el nivel de materias anuladas, debido a que en la malla antigua existen 212 anulaciones y en la malla nueva 198 anulaciones. Sin embargo, es conveniente realizar una revisión en los datos de la malla rediseñada.

Tabla 10: Retiro estudiantil Mayo 2015 a Septiembre 2017

\begin{tabular}{|c|c|c|c|}
\hline Período & $\begin{array}{l}\text { Estudiantes inscritos según Sistema } \\
\text { Nacional de Admisión }\end{array}$ & Nota 0 & $\begin{array}{l}\text { Equivalente de } \\
\text { retiro }\end{array}$ \\
\hline Mayo 2015 - Septiembre 2015 & 1682 & 410 & $24,38 \%$ \\
\hline Octubre 2015 - Febrero 2016 & 1943 & 400 & $20,59 \%$ \\
\hline Mayo 2016 - Septiembre 2016 & 2295 & 330 & $14,38 \%$ \\
\hline Octubre 2016 - Febrero 2017 & 2039 & 273 & $13,39 \%$ \\
\hline Mayo 2017 - Septiembre 2017 & 2376 & 256 & $10,77 \%$ \\
\hline
\end{tabular}

Fuente: Secretaria de Campus UPS Sede Guayaquil

Para analizar la tasa de retención se debe trabajar con los estudiantes inscritos de primer nivel de dos años atrás versus los que se encuentran en quinto nivel; para efectos de la carrera se trabajan con los datos proporcionados del Sistema Nacional de Admisión.

\section{Tabla 11: Tasa de retención Semestral 2014 - 2017}




\begin{tabular}{lcccc}
\hline Semestre Equivalente & $\begin{array}{c}\text { Estudiantes que } \\
\text { continúan después de 2 } \\
\text { años }\end{array}$ & $\begin{array}{c}\text { Estudiantes } \\
\text { admitidos 2 años } \\
\text { atrás }\end{array}$ & $\begin{array}{c}\text { Semestre de } \\
\text { origen }\end{array}$ & $\begin{array}{c}\text { Tasa de } \\
\text { retención } \\
\text { semestral }\end{array}$ \\
\hline $2014-2015$ & 78 & 228 & $2012-2013$ & $34,21 \%$ \\
$2015-2015$ & 255 & 537 & $2013-2013$ & $47,49 \%$ \\
$2015-2016$ & 80 & 196 & $2013-2014$ & $40,82 \%$ \\
$2016-2016$ & 261 & 670 & $2014-2014$ & $38,96 \%$ \\
$2016-2017$ & 199 & 463 & $2014-2015$ & $42,98 \%$ \\
$2017-2017$ & 239 & 809 & $2015-2015$ & $29,54 \%$ \\
\hline
\end{tabular}

Fuente: Secretaria de Campus UPS Sede Guayaquil

En el año 2015 toda la UPS vivió el mayor grado de deserción estudiantil, debido a que muchos estudiantes optaron por no matricularse debido a factores económicos y a una política de oyentes, la cual obligaba al estudiante a volver a repetir y aprobar una asignatura por cuarta vez, situación que generó mucho malestar a todo nivel. En el año 2017 se crea la política de actividades de remediación y examen de recuperación, la cual ha reducido la deserción y aumenta la retención estudiantil.

\section{Discusión}

Con el propósito de evitar la deserción y disminuir el índice de repitencia y al mismo tiempo mejorar la aprobación de cada materia y obtener un mejor uso de tutorías en el proceso de eficiencia terminal, proponemos acciones de mejora de carácter semestral enfocado al Criterio Estudiantes. Constituyen evidencias de esta actividad las siguientes: Informes de Gestión, Registros e Informes de tutorías académicas.

Tabla 12: Propuesta de Acciones de Mejora para mejorar eficiencia terminal

\begin{tabular}{|c|c|c|}
\hline Objetivo & Actividad & Táctica \\
\hline $\begin{array}{l}\text { Proponer alternativas para } \\
\text { evitar la deserción y pérdida del } \\
\text { semestre antes del examen final. }\end{array}$ & $\begin{array}{l}\text { Realizar una proyección } \\
\text { académica de los posibles } \\
\text { desertores en base a las notas } \\
\text { del Primer Interciclo. } \\
\text { Proponer estrategias para } \\
\text { ayudar a los estudiantes con } \\
\text { riesgo académico alto. }\end{array}$ & $\begin{array}{l}\text { Armar la estadística de las materias sujetas a } \\
\text { retiro académico después de los exámenes del } \\
\text { primer interciclo } \\
\text { Solicitar a los docentes que tengan paralelos } \\
\text { con riesgo académico alto planes remediales. }\end{array}$ \\
\hline $\begin{array}{l}\text { Constatar el cumplimiento de } \\
\text { las actividades propuestas por } \\
\text { los docentes con paralelos de } \\
\text { riesgo académico alto. }\end{array}$ & $\begin{array}{l}\text { Evidenciar el compromiso } \\
\text { de estudiantes y docentes de } \\
\text { los planes remediales en cada } \\
\text { asignatura. }\end{array}$ & $\begin{array}{l}\text { Solicitar a cada docente en vía digital el } \\
\text { modelo del trabajo propuesto de recuperación } \\
\text { en actividades remediales como tutorías, } \\
\text { lecciones, deberes, exposiciones, } \\
\text { investigaciones. }\end{array}$ \\
\hline
\end{tabular}

Elaborado por: Los autores

Para la obtención de mejores resultados, es relevante que todos los docentes publiquen en la primera semana de clases los cronogramas con las respectivas rúbricas, las mismas que determinan la metodología a llevarse en cada asignatura, lo cual permite al estudiante conocer los parámetros de evaluación desde el inicio del semestre. 
En el aprovechamiento se debe considerar como mínimo dos indicadores por cada actividad académica a ser evaluada. Los docentes aplican diversos tipos de evaluaciones con el fin de que el estudiante demuestre sus conocimientos aplicando las competencias adquiridas en el proceso de enseñanza - aprendizaje.

Cada semestre los docentes con cursos en niveles de riesgo académico deben presentar planes remediales señalando las diferentes técnicas que aplicaron para evitar la pérdida de materia a través de tutorías, lecciones, trabajos adicionales, estudio de casos, investigaciones, exposiciones, que permiten acompañamiento en el proceso del estudiante.

Esta alternativa permite un mayor acercamiento con estudiantes y docentes debido a las visitas áulicas que son más seguidas. Los temas propuestos en exámenes no deben ser concatenados para que los estudiantes no sean perjudicados y tengan la oportunidad de realizar otra pregunta en caso de no poder responder la pregunta original (Rodríguez, 2004).

\section{Conclusiones}

Las tutorías académicas acompañan al estudiante tanto al inicio de su carrera como en su culminación a través de la unidad de titulación.

Revisando sus conceptos, el alcance de las mismas se refleja en la Academia y en las cohortes porque brindan un servicio y un derecho que ayudan a mejorar la eficiencia terminal, por consiguiente permiten puntuar mejor a cada carrera dentro de la autoevaluación (Klenk, 1999; Beede \& Burnett, 1999; Ferrer, 2003).

Las tutorías deben continuar aplicándose para reducir la cantidad de reprobados, permitir que los estudiantes se nivelen y adelanten materia.

Es importante conocer la efectividad de las mismas para controlar y tratar de reducir el retiro estudiantil y la retención de las cohortes (Michavila \& García, 2003; Schroeder y Hurst, 1996)

\section{Bibliografía}

Aguilar. (01 de 01 de 2014). Repositorio Digital UPS. Obtenido de https://dspace.ups.edu.ec/bitstream/123456789/6889/1/UPS-QT05477.pdf

ALCON, E. ET AL. (2003). La tutoría y los nuevos modos de aprendizaje en la universidad. Madrid: CAM-Cátedra UNESCO

ALONSO CANO, C. (2000). Las nuevas funciones docentes y los nuevos medios. Barcelona: Facultad de Pedagogía de la Universidad de Barcelona.

AUTMENDI ET AL. (2003). Cómo diseñar materias y realizar tutorías en la formación "on line".Bilbao: Universidad de Deusto 
Balás, M. A. (21 de 01 de 2016). Seminario La acreditación como cultura de la evaluación de la calidad en Educación a distancia. Obtenido de http://reposital.cuaed.unam.mx:8080/jspui/handle/123456789/4749

Balás. (2016). Retiro Estudiantil Salesiano: ¿quién es culpable: la universidad, la metodología docente o el desempeño estudiantil? Innova Research. Obtenido de http://www.journaluidegye.com/magazine/index.php/innova/article/view/57

Beede, M. y Burnett, D. (Eds.) (1999), Planning for student services: Best practices for the 21st century, Michigan: Society for College and University Planning.

Ceaaces. (2017). Modelo genérico de Evaluación del entorno de aprendizaje. Obtenido de http://www.ceaaces.gob.ec/sitio/wp-content/uploads/2013/10/Modelo-Gen\%C3\%89ricoDe-Evaluaci\%C3\%93n-Del-Entorno-De-Aprendizaje-Carreras-Presenciales-YSemipresenciales-De-Las-Universidades-Y-Escuelas-Polit\%C3\%89cnicas-DelEcuador.pdf

CES. (01 de 01 de 2007). Consejo de Educación Superior. Obtenido de http://www.ces.gob.ec/doc/2-seminario/unidad-de-titulacion.pdf

FERRER, J. (2003). "La acción tutorial en la universidad". En MICHAVILAF. \& J. Garcíadelgado(eds.),La Tutoría y los Nuevos Modos de Aprendizaje en la Universidad. Madrid: CAM-Cátedra UNESCO

GARCÍA NIETO, N. (1996). "Los contenidos de la Función Tutorial". Revista Complutense de Educación, 7, 1.

Hower, T. (2007). Perfil de Egreso.

Klenk, S.W. (1999), Customer-based transformation, en Beede, M. y Burnett, D. (Eds.), Planning for sutent services: Best practices for the 21st century, Michigan: Society for College and University Planning.

MICHAVILA, F. \& J. Garcíadelgado(eds.) (2003). La Tutoría y los Nuevos Modos de Aprendizaje en la Universidad. Madrid: CAM-Cátedra UNESCO.

RAGA, J. T. (2003). "La Tutoría, Reto de una Universidad Formativa". En F. Michavila\& J. Garcíadelgado(eds.), La Tutoría y los Nuevos Modos de Aprendizaje en la Universidad.Madrid

RODRÍGUEZ ESPINAR, S. (coord.) (2004). Manual de tutoría universitaria. Barcelona: Octaedro/ICE-UB.

Schroeder, C.C., y Hurst, J.C. (1996), Designing learning environments that integrate curricular and concurricular experiencies, Journal of College Student Development , 37 (2), 174-181. 
Terenzini, P.T.; Springer, L.; Pascarella, E.T. y Nora, A. (1995), Influences affecting the development of students' critical thinking skills, Research in Higher Education , 36, 23 39. 